\title{
Aromatic Amidines as Fluorogenic Reagents for Reducing Carbohydrates
}

\author{
Masaaki Kal*, Kazuhiko Tamura* Masatoshi YAmaguchi** and Yosuke OHKURA* \\ *Faculty of Pharmaceutical Sciences, Kyushu University, \\ Maidashi, Fukuoka 812 \\ **Faculty of Pharmaceutical Sciences, Fukuoka University, \\ Nanakuma, Fukuoka 814-01
}

\begin{abstract}
Aromatic amidines such as 4-methoxybenzamidine, benzamidine, 3,4-dimethoxybenzamidine, 2-amidinonaphthalene and 4-amidinopyridine give fluorescences when reducing carbonhydrates are heated in an alkaline medium with these amidines for a few minutes. Of the amidines tested, 4-methoxybenzamidine and benzamidine are favorable fluorogenic reagents for reducing carbohydrates. Both the aromatic amidines permit the fluorometric determination of reducing carbohydrates with rapid and simple procedure; reducing carbohydrates tested can be determined at concentrations as low as $0.06-2.25 \mathrm{nmol} \mathrm{ml}^{-1}$ and $0.09-2.40 \mathrm{nmol} \mathrm{ml}^{-1}$ by the methods with 4-methoxybenzamidine and benzamidine, respectively.
\end{abstract}

Keywords Fluorometric determination, reducing carbohydrate, aromatic amidine, 4-methoxybenzamidine, benzamidine

Many fluorogenic reagents have been proposed for analyses of reducing carbohydrates. The reagents are classified into two groups in regard to their reaction conditions. One is the reagents such as 5-hydroxytetralone', $o$-phenylenediamine ${ }^{2}$, resorcinol ${ }^{3}$ and $N$-(1-naphthyl)ethylenediamine ${ }^{4}$, which all require strongly acidic conditions, and thus most of saccharides are decomposed by acidic hydrolysis. The reagents of the other group are ethylenediamine ${ }^{5}$, taurine ${ }^{6}$, malonamide $^{7}, 2$-ethanolamine ${ }^{8}, 2$-cyanoacetamide ${ }^{9}$ and arginine $e^{10}$, which allow fluorometric determination of reducing carbohydrates in a weakly alkaline or neutral medium with prolonged heating time longer than 30 $\min$.

During a survey of various amidino or guanidino compounds as the reagents for fluorometric determination of carbohydrates, we found that several aromatic amidines provide fluorescences for reducing carbohydrates under alkaline conditions, as a different type from the reagents mentioned above. The survey was carried out, expecting that ketoalcoholic moiety of carbohydrates might react with amidino and/or guanidino groups to produce fluorescent imidazole derivative(s) under alkaline conditions, since guanidino compounds react with benzoin having a ketoalcoholic moiety to form fluorescent diphenylimidazole derivatives under alkaline conditions. ${ }^{11-13}$ With similar view, Seki and Yamaguch ${ }^{14}$ have recently developed a fluorometric determination of 17-hydroxycorticosteroids by high performance liquid chromatography using benzamidine as a fluorogenic reagent in the post-column derivatization system.

In this paper, we discuss fluorescent reactivities of various amidino or guanidino compounds to reducing carbohydrates, and further describe optimum reaction conditions for the fluorometric determination of reducing carbohydrates with 4-methoxybenzamidine or benzamidine which gave good results as a fluorogenic reagent among the compounds tested. D-Glucose was employed as a representative compound for the investigations.

\section{Experimental}

\section{Reagents}

Deionized and distilled water was used throughout. Benzamidine was purchased from Aldrich Chemical Co., Inc., (Milwaukee, WIS, USA). 4-Methoxybenzamidine was synthesized according to the method of Pinner. ${ }^{15}$ 3,4-Dimethoxybenzamidine, 2-amidinonaphthalene and 4-amidinopyridine were kindly supplied by Dr. Kina (Dojindo Laboratory, Kumamoto, Japan). All other chemicals were of reagent grade.

\section{Apparatus}

Fluorescence spectra and intensities were measured with a Hitachi MPF-4 spectrofluorometer in $10 \times 10 \mathrm{~mm}$ 
quartz cells; spectral bandwidths of $10 \mathrm{~nm}$ were used in both the excitation and emission monochromators. The fluorescence spectra, and excitation and emission maxima are uncorrected.

\section{Analytical procedure for reducing carbohydrate}

To $1.0 \mathrm{ml}$ of a sample solution in a test tube, $1.0 \mathrm{ml}$ of an aqueous solution of aromatic amidine (30 $\mathrm{mM} \mathrm{4}$ methoxybenzamidine or benzamidine) and $1.0 \mathrm{ml}$ of $1.0 \mathrm{M}$ potassium hydroxide solution are added. The mixture is heated in a boiling water-bath for $3 \mathrm{~min}$ for 4-methoxybenzamidine or for 2 min for benzamidine, and then cooled in ice-water. The reagent blank is prepared in the same way except that $1.0 \mathrm{ml}$ of the sample solution is replaced with $1.0 \mathrm{ml}$ of water.

The fluorescence intensity is measured at $470 \mathrm{~nm}$ emission against $310 \mathrm{~nm}$ excitation for the reagent of 4-methoxybenzamidine, or measured at $470 \mathrm{~nm}$ emission against $365 \mathrm{~nm}$ excitation for benzamidine.

\section{Results and Discussion}

\section{Screening of amidino or guanidino compounds as fluorogenic reagent}

Fluorescence from reducing carbohydrate occurs in reactions with aromatic amidines tested (eg. benzamidine, 4-methoxybenzamidine, 3,4-dimethoxybenzamidine, 2-amidinonaphthalene and 4-amidinopyridine) under alkaline conditions with heating, but neither with aliphatic amidine (acetamidine) nor guanidino compounds tested (eg. methylguanidine and phenylguanidine). Table 1 shows the fluorescence intensities from D-glucose and those of the reagent blanks, obtained with the aromatic amidines. 4-Methoxybenzamidine gives the highest intensity for equimolar amount of $D-$ glucose, though benzamidine also gives fluorescence enough to determine the reducing carbohydrate at subnano mole level. The aromatic amidines other than 4methoxybenzamidine and benzamidine are not favorable reagents because of high values of their reagent blanks and besides of low yield of fluorescence from D-glucose. Therefore, 4-methoxybenzamidine and benzamidine were investigated further to establish suitable reaction conditions for analytical method of reducing carbohydrates.

On the other hand, Mikami and Ishida ${ }^{10}$ have recently reported that arginine gives fluorescence in the reaction with reducing carbohydrates under neutral conditions similar to those with ethanolamine ${ }^{8}$, i.e. heating in a borate buffer ( $\mathrm{pH} \mathrm{7.5)}$ at higher temperature than $120^{\circ} \mathrm{C}$. Thus, guanidino compounds tested, phenylguanidine and methylguanidine offer fluorescence for Dglucose provided that the reaction is performed in a neutral borate buffer with heating at $145^{\circ} \mathrm{C}$; but the intensity was extremely low in comparison with that given by benzamidine. On the contrary, the aromatic amidines tested as well as acetamidine do not permit any development of fluorescence from D-glucose in a weakly alkaline or neutral medium (phosphate or borate buffer), even with heating at a high temperature $\left(145^{\circ} \mathrm{C}\right)$.

\section{Determination of D-glucose with 4-methoxyben- zamidine or benzamidine}

With 4-methoxybenzamidine, the fluorescence spectra produced from $\mathrm{D}$-glucose show an emission maximum at $470 \mathrm{~nm}$ with two excitation maxima at 310 and 365 $\mathrm{nm}$ (Fig. 1); the intensity at $470 \mathrm{~nm}$ emission with irradiation at $310 \mathrm{~nm}$ is approximately twice as great as that with irradiation at $365 \mathrm{~nm}$.

On the other hand, benzamidine gives two different fluorescence spectra for $\mathrm{D}$-glucose depending on the reaction time (Fig. 2). When the reaction time is relatively short (approximately less than $10 \mathrm{~min}$, see in Fig. 4), the fluorescence which has an emission maximum at $470 \mathrm{~nm}$ (Fig. 2, $b_{1}$ ) and two excitation maxima at 295 and $365 \mathrm{~nm}$ (Fig. 2, $\mathrm{a}_{1}$ ), is produced; the intensity at $470 \mathrm{~nm}$ emission with irradiation at $365 \mathrm{~nm}$ is measured for the determination of reducing carbohydrates. For further reaction periods, another fluorescence occurs which shows an emission and excitation maxima at 370 and $300 \mathrm{~nm}$, respectively (Fig. $2, a_{3}$ and $b_{3}$ ). Such two different fluorescent species are also observed when other reducing carbohydrates (listed in Table 2) are treated with benzamidine in the same way. From these observations, at least two fluorescent

Table 1 Fluorescence intensities produced by reactions of D-glucose with aromatic amidines and their reagent-blank values

\begin{tabular}{|c|c|c|c|c|}
\hline \multirow{2}{*}{ Aromatic amidine } & \multicolumn{2}{|c|}{ R. F. I. } & \multirow{2}{*}{$\begin{array}{c}\text { Excitation } \\
\text { maximum/ } \\
\text { nm }\end{array}$} & \multirow{2}{*}{$\begin{array}{c}\text { Emission } \\
\text { maximum }\end{array}$} \\
\hline & Testb & Blank $^{\mathbf{b}}$ & & \\
\hline Benzamidine & 100 & 1.2 & 365 & 470 \\
\hline 4-Methoxybenzamidine & 550 & 5.0 & 310 & 470 \\
\hline 3,4-Dimethoxybenzamidine & 72 & 17 & 330 & 470 \\
\hline 2-Amidinonaphthalene & 36 & 61 & 355 & 480 \\
\hline 4-Amidinopyridine & 5 & 32 & 360 & 455 \\
\hline
\end{tabular}

a, The relative fluorescence intensity obtained by the reaction of $\mathrm{D}$-glucose with benzamidine was taken as 100 . b, Portions $(1.0 \mathrm{ml})$ of $30 \mathrm{nmol} \mathrm{ml-1} \mathrm{D}$-glucose solution and water for test and blank, respectively, were treated according to the analytical procedure, but the reaction time was 2 min for benzamidine, 2-amidinonaphthalene and 4-amidinopyridine and $3 \mathrm{~min}$ for the others. The concentration of each aromatic amidine was $30 \mathrm{mM}$. 
products must be formed for a reducing carbohydrate by the reaction with benzamidine.

In the reaction of D-glucose with 4-methoxybenzamidine, the fluorescence dose not arise at a temperature less than $23^{\circ} \mathrm{C}$, however, a temperature higher than approximately $70^{\circ} \mathrm{C}$ allows fluorescence to develop rapidly (Fig. 3). The maximum intensity at 100

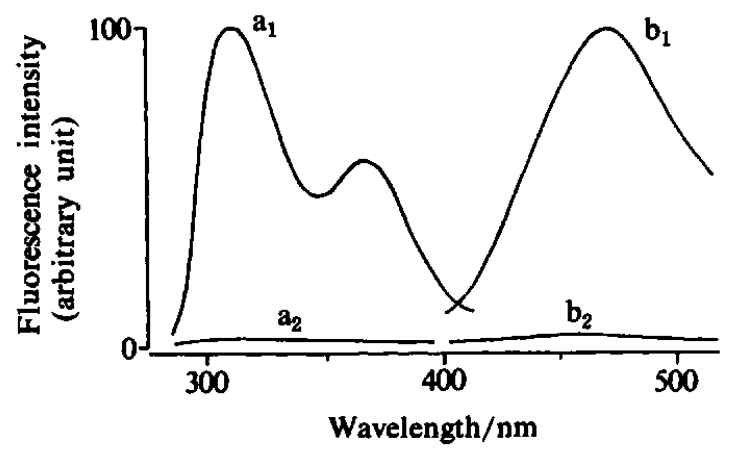

Fig. 1 Excitation and emission spectra of the final reaction mixture of $\mathrm{D}$-glucose with 4-methoxybenzamidine.

$a$, excitation spectra; $b$, emission spectra. $a_{1}$ and $b_{1}$ : a portion $(1.0 \mathrm{ml})$ of $30 \mathrm{nmol} \mathrm{ml}^{-1} \mathrm{D}-\mathrm{glucose}$ solution was treated by the procedure recommended. $a_{2}$ and $b_{2}$ : reagent blank corresponding to $a_{1}$ and $b_{1}$.
${ }^{\circ} \mathrm{C}$ is achieved by heating for $3 \mathrm{~min}$.

Similar results are also observed in the reaction with benzamidine (Fig. 4), though another fluorescence, with emission and excitation maxima at 370 and $300 \mathrm{~nm}$, respectively, is produced with prolonged reaction time

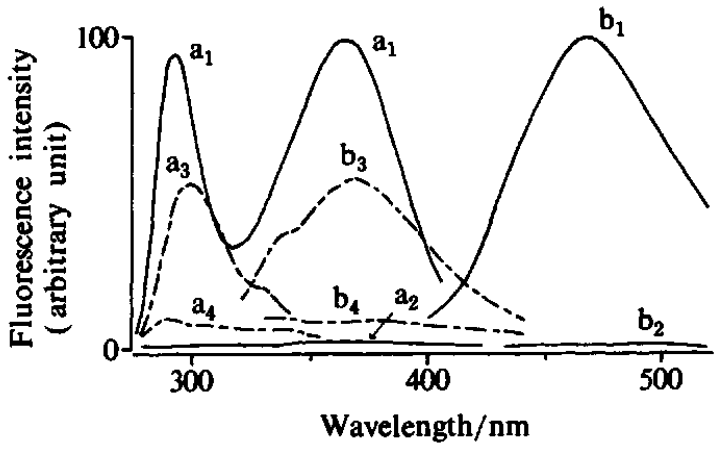

Fig. 2 Excitation and emission spectra of the final reaction mixture of $\mathrm{D}$-glucose with benzamidine.

$a$, excitation spectra; $b$, emission spectra. $a_{1}$ and $b_{1}$ : a portion $(1.0 \mathrm{ml})$ of $30 \mathrm{nmol} \mathrm{ml-1}$ D-glucose solution was treated as recommended. $a_{2}$ and $b_{2}$ : reagent blank corresponding to $a_{1}$ and $b_{1}, a_{3}$ and $b_{3}$ : a portion $(1.0 \mathrm{ml}$ ) of $30 \mathrm{nmol} \mathrm{ml}^{-1} \mathrm{D}$-glucose solution was treated as recommended, but the reaction time was $20 \mathrm{~min}$. $a_{4}$ and $b_{4}$ : reagent blank corresponding to $a_{3}$ and $b_{3}$.

Table 2 Fluorescence intensities obtained by the reactions of various reducing carbohydrates with 4-methoxybenzamidine or benzamidine, and their lower limits of determination

\begin{tabular}{|c|c|c|c|c|}
\hline \multirow[b]{2}{*}{ Reducing carbohydrate ${ }^{a}$} & \multicolumn{2}{|c|}{ 4-Methoxybenzamidine } & \multicolumn{2}{|c|}{ Benzamidine } \\
\hline & R. F. I. ${ }^{\mathbf{b}}$ & $\begin{array}{l}\text { Lower limitc } \\
\text { nmol } \mathrm{ml}^{-1}\end{array}$ & R.F. I. ${ }^{\mathbf{b}}$ & $\begin{array}{l}\text { Lower limitc } \\
\text { nmol ml }{ }^{-1}\end{array}$ \\
\hline D-Glucose & 550 & 0.27 & 100 & 0.36 \\
\hline D-Galactose & 374 & 0.40 & 69 & 0.52 \\
\hline D-Mannose & 480 & 0.31 & 92 & 0.39 \\
\hline D-Fructose & 725 & 0.20 & 106 & 0.34 \\
\hline 2-Deoxy-D-glucose & 83 & 1.80 & 16 & 2.25 \\
\hline D-Glucosamine & 105 & 1.42 & 17 & 2.12 \\
\hline D-Galactosamine & 204 & 0.73 & 34 & 1.06 \\
\hline$N$-Acetylglúcosamine & 325 & 0.46 & 49 & 0.73 \\
\hline L-Rhamnose & 253 & 0.59 & 36 & 1.00 \\
\hline L-Fucose & 220 & 0.68 & 47 & 0.77 \\
\hline D-Glucose-6-phosphate & 330 & 0.45 & 67 & 0.54 \\
\hline D-Glucuronolactone & 517 & 0.29 & 90 & 0.40 \\
\hline$N$-Acetylneuramic acid & 115 & 1.29 & 15 & 2.40 \\
\hline D-Ribose & 385 & 0.39 & 57 & 0.63 \\
\hline D-Arabinose & 360 & 0.42 & 58 & 0.62 \\
\hline D-Xylose & 457 & 0.33 & 70 & 0.51 \\
\hline D-Xylulose & 535 & 0.28 & 94 & 0.38 \\
\hline 2-Deoxy-D-ribose & 66 & 2.25 & 15 & 2.40 \\
\hline D-Erythrose & 2400 & 0.06 & 403 & 0.09 \\
\hline DL-Glyceraldehyde & 2330 & 0.06 & 263 & 0.14 \\
\hline Maltose & 567 & 0.26 & 100 & 0.36 \\
\hline Gentiobiose & 583 & 0.25 & 110 & 0.32 \\
\hline Lactose & 320 & 0.47 & 68 & 0.53 \\
\hline
\end{tabular}

a, Portion $(1.0 \mathrm{ml})$ of $30 \mathrm{nmol} \mathrm{ml}^{-1}$ solution of each compound was treated with 4-methoxybenzamidine or benzamidine according to the procedure recommended. $\mathrm{b}$, The relative fluorescence intensity obtained by the reaction of D-glucose with benzamidine was taken as 100 . c, Defined as the concentration which gives a fluorescence intensity twice the blank. 


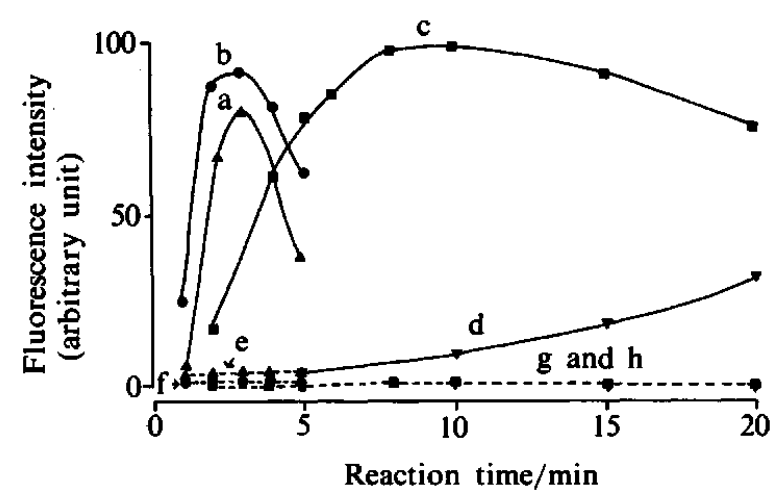

Fig. 3 Effects of reaction time and temperature on the fluorescence development in the reaction of $D$ glucose with 4-methoxybenzamidine.

Portions $(1.0 \mathrm{ml})$ of $30 \mathrm{nmol} \mathrm{ml-1}$ D-glucose solution were treated as recommended for various reaction times at different reaction temperatures. a, $145^{\circ} \mathrm{C} ; \mathrm{b}, 100^{\circ} \mathrm{C}$; c, $70^{\circ} \mathrm{C} ; \mathrm{d}, 50^{\circ} \mathrm{C}$. e-h: reagent blanks corresponding to $\mathrm{a}-\mathrm{d}$, respectively.

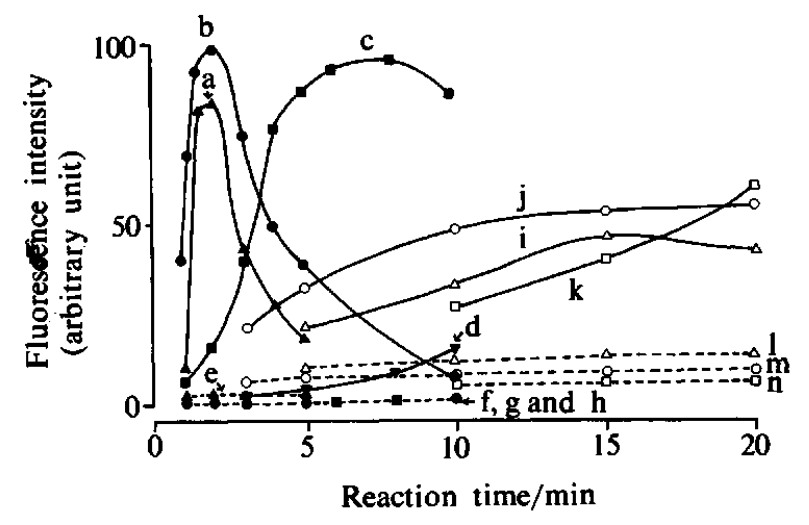

Fig. 4 Effects of reaction time and temperature on the fluorescence development in the reaction of $D^{-}$ glucose with benzamidine.

Portions $(1.0 \mathrm{ml})$ of $30 \mathrm{nmol} \mathrm{ml}^{-1} \mathrm{D}$-glucose solution were treated as recommended for various reaction times at different reaction temperatures. $a$ and $i, 145^{\circ} \mathrm{C} ; b$ and $j$, $100^{\circ} \mathrm{C}$; c and $\mathrm{k}, 70^{\circ} \mathrm{C}$; d, $50^{\circ} \mathrm{C}$. a-h: measured at 470 $\mathrm{nm}$ emission with irradiation at $365 \mathrm{~nm}$. $\mathrm{i}-\mathrm{n}$ : measured at $370 \mathrm{~nm}$ emission with irradiation at $300 \mathrm{~nm}$. $e-\mathrm{h}$ and $1-\mathrm{m}$ : reagent blanks corresponding to $a-d$ and $i-k$, respectively.

as demonstrated in Fig. 2. The latter fluorescence is apparently less intense, over the reaction times examined at any temperature, than the former one with maximum intensity at $470 \mathrm{~nm}$ emission and $365 \mathrm{~nm}$ excitation.

Consequently, to obtain an intense fluorescence from reducing carbohydrate, a short reaction time at $100^{\circ} \mathrm{C}(3$ $\min$ for 4-methoxybenzamidine and $2 \mathrm{~min}$ for benzamidine) is selected in the analytical procedure. In this case, the final reaction mixture must be cooled quickly in ice-water at the end of the time in order to

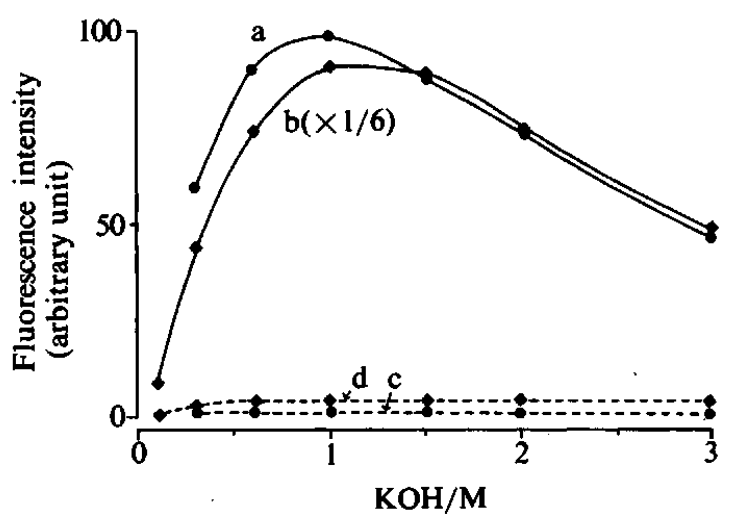

Fig. 5 Effect of potassium hydroxide concentration on the fluorescence development in the reaction of $\mathrm{D}$-glucose with benzamidine and 4-methoxybenzamidine.

Portions $(1.0 \mathrm{ml})$ of $30 \mathrm{nmol} \mathrm{ml}^{-1} \mathrm{D}$-glucose solution were reacted with the reagent of (a) benzamidine or (b) 4methoxybenzamidine according to the procedure recommended with various concentrations of potassium hydroxide. $c$ and $d$ : reagent blanks corresponding to $a$ and $b$, respectively.

stop the reaction. This treatment is also an aid in stabilizing the produced fluorescence for at least $1 \mathrm{~h}$. When the reaction mixture is allowed to stand at room temperature $\left(23^{\circ} \mathrm{C}\right)$, the fluorescence intensity decreases gradually with time at the rate of about $20 \% \mathrm{~h}^{-1}$ in both the reactions.

For developing fluorescence from reducing carbohydrates, both the reactions with 4-methoxybenzamidine and benzamidine should be carried out in an aqueous potassium or sodium hydroxide solution. The maximum intensity from D-glucose is attained by $1.0 \mathrm{M}$ potassium hydroxide in both the reactions (Fig. 5). Sodium hydroxide at the concentration of $1.0 \mathrm{M}$ gives slightly weak fluorescence, approximately $80 \%$ of that given by $1.0 \mathrm{M}$ potassium hydroxide, in each reaction.

A concentration of benzamidine at $25 \mathrm{mM}$ or greater, or of 4-methoxybenzamidine at $30 \mathrm{mM}$ or greater provides a maximum constant fluorescence from $D$-glucose, but higher concentrations of each reagent result in increasing values of the reagent blank; $30 \mathrm{mM}$ each solution was used in the analytical procedure.

The calibration graphs obtained by both the analytical procedures with 4-methoxybenzamidine and benzamidine were linear over the concentration range 0.1$200 \mathrm{nmol} \mathrm{ml} \mathrm{m}^{-1}$ of D-glucose. The precision was established by repeating 20 analyses at the same time on $30 \mathrm{nmol} \mathrm{ml}^{-1} \mathrm{D}$-glucose. The coefficients of variation were 1.9 and $2.1 \%$ for the methods with 4methoxybenzamidine and benzamidine, respectively.

Fluorescences from other carbohydrates in the reaction with 4-methoxybenzamidine or benzamidine

All of the reducing carbohydrates tested can fluoresce under the reaction conditions established for both the 
reagents. Their relative fluorescence intensities and the minimum determinable concentrations are listed in Table 2. The excitation and emission spectra of the fluorescences from these reducing carbohydrates are very similar in shape and maxima to those of the fluorescence from D-glucose and are not characteristic of individual carbohydrates, in each case using 4-methoxybenzamidine or benzamidine.

On equimolar basis, 4-methoxybenzamidine gives fluorescence intensities approximately 5.5 times higher than those given by benzamidine for all the reducing carbohydrates tested, though its reagent-blank value is also 4 times higher than that of benzamidine. Among the reducing carbohydrates tested, 2-deoxyaldoses and aminosugars fluoresce rather weakly, and D-erythrose (a tetrose) and DL-glyceraldehyde (a triose) fluoresce intensely in each reaction. In consequence, lower determinable limits of the carbohydrates with 4-methoxybenzamidine and benzamidine are given by $0.06-2.25 \mathrm{nmol}$ $\mathrm{ml}^{-1}$ and 0.09-2.40 $\mathrm{nmol} \mathrm{ml} \mathrm{m}^{-1}$, respectively.

Non-reducing carbohydrates including glycosides, alditols and aldonic acids such as sucrose, raffinose, stachyose, $\alpha$-methyl-D-glucoside, D-glucose-1-phosphate, D-sorbitol, D-inositol, D-glucosaccharo-1,4-lactone and neuramic acid- $\boldsymbol{\beta}$-methylglucoside give no fluorescence, except that gluconic acid gives fluorescences with 66 and $16 \%$ intensities of that given by an equimolar amount of D-glucose in the reactions with 4methoxybenzamidine and benzamidine, respectively.

\section{Feature of the reagents}

4-Methoxybenzamidine and benzamidine permit fairly selective determination of reducing carbohydrates since these reagents do not provide fluorescences for 17 different $\mathrm{L}-\boldsymbol{\alpha}$-amino acids, aldehydes (formaldehyde, acetaldehyde and benzaldehyde), keto acids ( $\alpha-$ ketoglutaric acid, phenylpyruvic acid and $p$-hydroxyphenylpyruvic acid), carboxylic acids (acetic acid and formic acid) and nucleosides (adenosine and cytidine). These reagents also offer advantage of rapidity in reaction over other fluorogenic reagents so far reported for the determination of reducing carbohydrates. Thus, both the reagents may be successfully used for automatic detection system after chromatographic separation; 4-methoxybenzamidine in paticular will be the greater reagent in the system because it gives very high yield of fluorescence for reducing carbohydrates. This work is in progress in our laboratory.

The chemical structure of fluorescent product(s) in the reaction of reducing carbohydrates with 4methoxybenzamidine or benzamidine remains unknown. The further study should be required for the mechanism of the fluorescence reactions with these aromatic amidines.

We wish to thank Dr. K. Kina, Dojindo Laboratory, for the generous donation of benzamidine derivatives.

\section{References}

1. T. Momose and Y. Ohkura, Chem. Pharm. Bull., 7, 31 (1959).

2. J. C. Towne and J. E. Spinker, Anal. Biochem., 35, 211 (1963).

3. C. G. Rodgers, C. W. Chambers and N. A. Clark, Anal. Chem., 38, 1851 (1966).

4. N. Bounias, Anal. Biochem., 106, 291 (1980).

5. S. Honda, K. Kakimoto, K. Suda, K. Kakehi and K. Takiura, Anal. Chim. Acta, 70, 133 (1974).

6. T. Kato and T. Kinoshita, Chem. Pharm. Bull., 26, 1291 (1978).

7. S. Honda, Y. Matsuda, M. Terao and K. Kakehi, Anal. Chim. Acta, 108, 421 (1979).

8. T. Kato and T. Kinoshita, Anal. Biochem., 106, 238 (1980).

9. S. Honda, Y. Matsuda, M. Takahashi, K. Kakehi and S. Ganno, Anal. Chem., 52, 1079 (1980).

10. H. Mikami and Y. Ishida, Bunseki Kagaku, 32, E207 (1983).

11. Y. Ohkura and M. Kai, Anal. Chim. Acta, 106, 89 (1979).

12. M. Kai, M. Yamaguchi and Y. Ohkura, Anal. Chim. Acta, 120, 411 (1980).

13. M. Kai, M. Miura, K. Kohashi and Y. Ohkura, Chem. Pharm. Bull., 29, 1115 (1981).

14. T. Seki and Y. Yamaguchi, J. Chromatogr., 305, 188 (1984).

15. A. Pinner, Ber., 16, 1654 (1883); 17, 178 (1884).

(Received September 1, 1984)

(Accepted October 22, 1984) 